\title{
PIPELINE OPTIMIZATION DESIGN BASED ON ADJOINT SOLVER
}

\author{
Da-guang Li ${ }^{1, \text { a) }}$ Ying Zhang ${ }^{2, b)}$ \\ 1 Department of Hydraulic Engineering, Tianjin Agricultural University \\ 2 Tianjin Petroleum Vocational and Technical College,301607 China \\ a)253175275@qq.com b)32232846@qq.com
}

\begin{abstract}
Adjoint optimize solver is a discrete optimization algorithm based on gradient optimization method. It has higher efficiency and lower requirement on engineering experience in comparison with traditional engineering optimization method, and has been gradually applied in every engineering industry. In this thesis, the application of adjoint optimize solver in pipeline design is focused.
\end{abstract}

Keywords: Adjoint, design, pipeline

\section{Introduction}

The propose of adjoint equations is to reduce the amount of design variables in equation solving then reduce the calculation quantity. The adjoint solver is based on gradient optimization method, which is first time used in fluid mechanics by Pironneau[1], and then developed into discrete optimization algorithm with the rapid development of computer science. The following paragraphs illustrate the theory systems[2].

Here $\underline{c}$ is used to represent the design variable, and the flow variable in every discrete grid is $\underline{q}^{v}$; the residual is $R$; the objective function is $J$, then the discrete equations will be:

$$
\begin{gathered}
R_{i}^{u}\left(\underline{q}^{0}, \underline{q}^{1}, \ldots . . \underline{q}^{M-1} ; \underline{c}\right)=0 \\
J=J\left(\underline{q}^{0}, \underline{q}^{1}, \ldots . . \underline{q}^{M-1} ; \underline{c}\right)
\end{gathered}
$$

Where $i=0, \ldots . . L-1 ; u=0, \ldots . . . M-1 ; M$ indicates the quantity of girds in the model while $L$ means the dimension of the vector.

When the design variable changes slightly, the objective function and the residual will follow the equation:

$$
\frac{\partial R_{i}^{u}}{\partial q_{j}^{v}} \delta q_{j}^{v}=-\left.\frac{\partial R_{i}^{u}}{\partial c_{j}}\right|_{q} \delta c_{j}
$$

Where $\delta c_{j}$ means the variation of design variable and $\delta q_{j}^{v}$ represent the flow parameter variation caused by the change of design variable.

In the same time, the objective function will change correspondingly, and their relationship is as follow:

$$
\partial J=\frac{\partial J}{\partial q_{j}^{v}} \delta q_{j}^{v}+\left.\frac{\partial J}{\partial c_{j}}\right|_{q} \delta c_{j}
$$

It is obvious from the equation, every time the design variable $\underline{c}$ changes, the variation of flow parameter $\delta q_{j}^{v}$, will be calculated then. As a calculation with large number of design variables, the flow parameter will be a great computational burden. In the face of this, the adjoint matrix will be adopted in this thesis and adjoint equations in control theory will be used to solve this problem. The equation is as follow: 
Assume the adjoint equation is:

$$
\left[\tilde{q}_{i}^{u} \frac{\partial R_{i}^{u}}{\partial q_{j}^{v}}\right] \delta q_{j}^{v}=-\left.\tilde{q}_{i}^{u} \frac{\partial R_{i}^{u}}{\partial c_{j}}\right|_{q} \delta c_{j}
$$

$$
\tilde{q}_{i}^{u} \frac{\partial R_{i}^{u}}{\partial q_{j}^{v}}=\frac{\partial J}{\partial q_{j}^{v}}
$$

Then the gradient of objective function will be:

$$
\delta J=\frac{\partial J}{\partial q_{j}^{v}} \delta q_{j}^{v}+\left.\frac{\partial J}{\partial c_{j}}\right|_{q} \delta c_{j}=\tilde{q}_{i}^{u} \frac{\partial R_{i}^{u}}{\partial q_{j}^{v}} \delta q_{j}^{v}+\left.\frac{\partial J}{\partial c_{j}}\right|_{q} \delta c_{j}=\left\{\left.\frac{\partial J}{\partial c_{j}}\right|_{q}-\left.\tilde{q}_{i}^{u} \frac{\partial R_{i}^{u}}{\partial c_{j}}\right|_{q}\right\} \delta c_{j}
$$

It is shown with the introduction of adjoint matrix, the flow parameter $\delta q_{j}^{v}$ eliminated after deduction. The equation is turned into a function that objective function changes with design variable, which results in much higher efficiency.

\section{The application of adjoint solver in pipeline optimazition}

In traditional optimization method for pressure drop property, if CAD software is used for optimization, the repeated modeling and calculating required will in a great extent increase the optimization time of the air duct, and due to the irregular unstructured grid and boundary layer, in the presence of alone by software for optimum design of deformation of the and staggered grid, failure model of risk, the optimization of air duct has greater constraints. Adjoint solver is success to avoid the emergence of the above problems, and the application of the case is as follows[3,4].

(1). Adjoint solver is not bounded by the engineering experience and has larger optimizing space

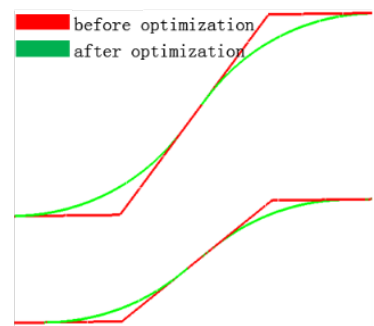

Engineering experience
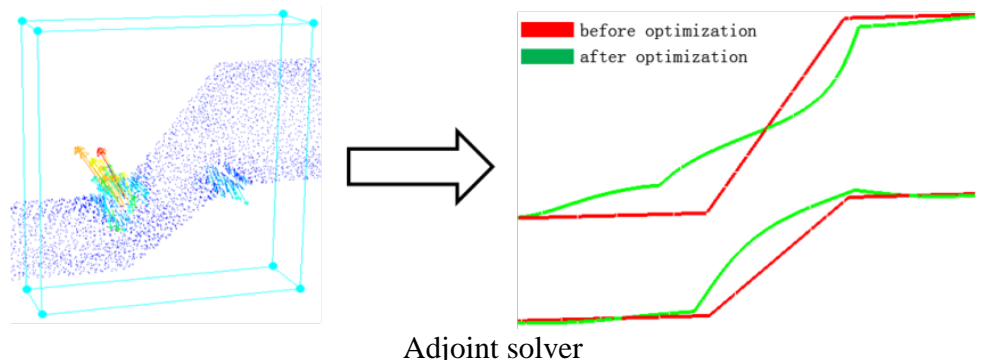

Figure 1.1 Different shapes of pipeline with two different optimizing methods

Figure 1.1 shows the comparison of sections obtained from different optimizing methods. The figure shows that traditional optimization method is mostly based on the original shape of the pipeline and smooth transition around the corner, while the adjoint solver optimization is based on the shape of the sensitivity of the air duct pressure drop and morph deformation. Comparing with traditional engineering experience, the adjoint solver is less affected by the original 
shape and has larger shape deformation space. For the malformation places on pipeline, CAD software is used to smooth process when the final shape is obtained.

(2). Adjoint solver has better optimize performance and lower dependence on engineering experience

Table 1.1 Pressure drop (Pa) comparison

\begin{tabular}{cccc}
\hline & $\begin{array}{l}\text { Total pressure } \\
\text { on inlet }\end{array}$ & $\begin{array}{l}\text { Total pressure } \\
\text { on outlet }\end{array}$ & Pressure drop \\
\hline Original & 110.68 & 16.56 & 94.12 \\
\hline Traditional method & 62.10 & 11.52 & 50.58 \\
\hline Adjoint solver & 44.88 & 14.12 & 30.76 \\
\hline
\end{tabular}

From Table 1.1 it can be concluded with adjoint solver optimization the pipeline will have the smallest pressure drop. With traditional method, different optimal pipeline will be obtained by different engineer. But for less experienced engineers, adjoint optimization method will bring great convenience. For more complex model, adjoint solver has good effectadn can also reduce the number of models and time.

\section{Application of adjoint solver in a specific design}

Now the duct shown in Figure1.2 is focused to minimize its pressure drop. Mass flow rate from inlet is $0.204 \mathrm{Kg} / \mathrm{s}$ and outlets are set pressure outlet where static pressure is 0 . Pressure drop of the original duct is shown in table 1.2.

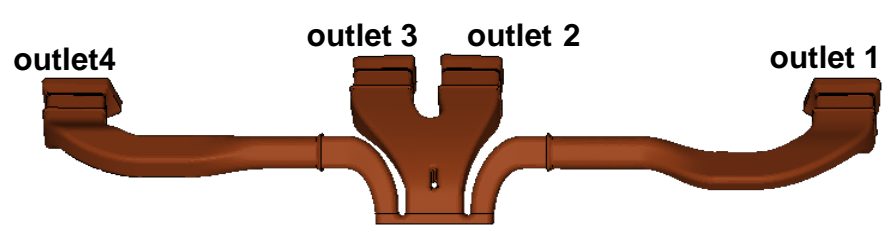

Figure 1.2 air duct model

Table 1.2 Pressure drop of original duct

\begin{tabular}{llll}
\hline $\begin{array}{l}\text { Total pressure } \\
\text { on inlet }\end{array}$ & \multicolumn{2}{l}{$\begin{array}{l}\text { Total pressure } \\
\text { on outlet }\end{array}$} & Pressure drop \\
\hline & & 138.26 & 114.08 \\
\hline
\end{tabular}

Form Table 1.2 it can be seen the duct has very large pressure drop and it is difficult to achieve the target performance. So it is necessary to optimize its pressure drop property.

After several times of traditional method optimization, the optimized duct is shown in Figure 1.3.

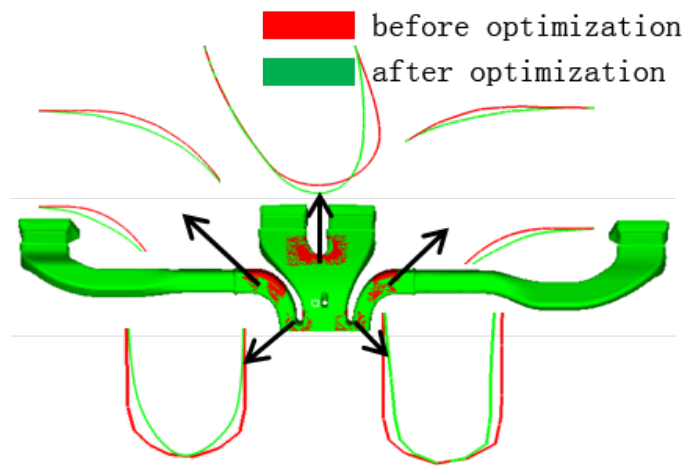

Figure 1.3 Comparison between shapes before and after traditional optimize 
To use adjoint solver method to optimize the air duct pressure drop, the first step is the shape sensitivity analysis for pressure drop, and then use morph function according to the shape sensitivity to deform the shape, and finally smooth the shape of the air duct. The optimization result is shown in Figure 1.4.

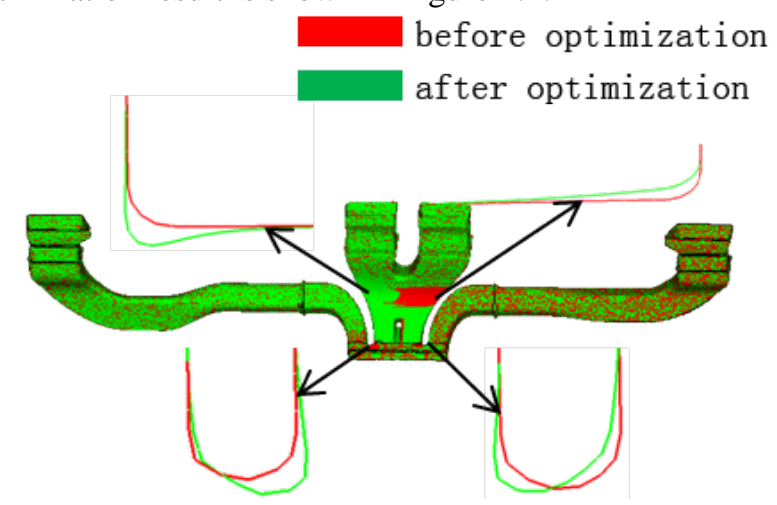

Figure 1.4 Comparison of shapes before and after optimization

Figure 1.5 displays the streamlines of original shape, traditional method optimized shape and adjoint solver optimized shape. From the streamlines it's known with adjoint solver optimized shape has least vertex in the duct and lower velocity on narrow sections, which contribute to the smaller pressure drop.

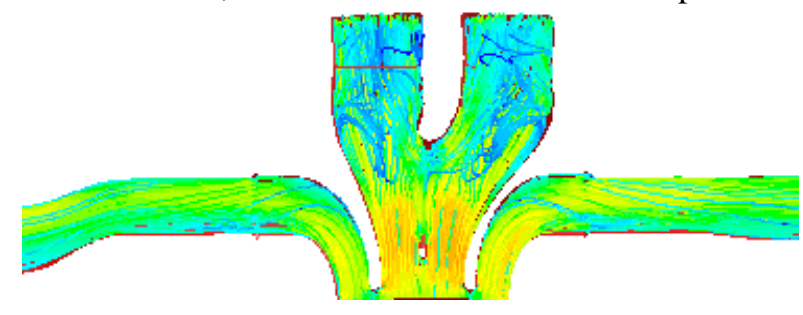

(a)Streamlines in original shape

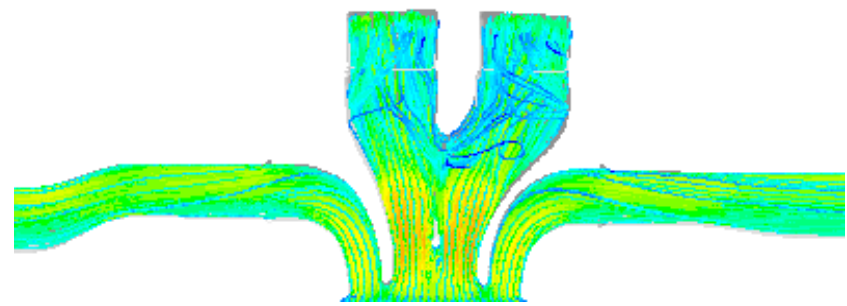

(b) Streamlines in traditional method optimized shape

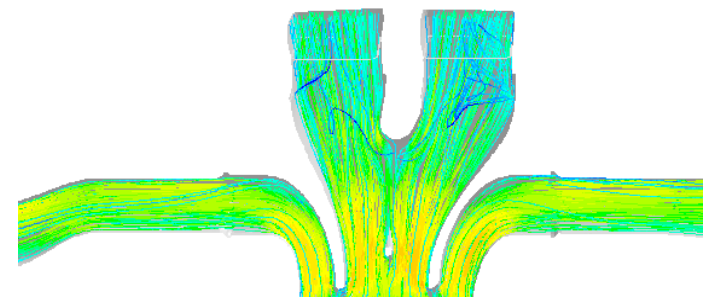

(c) Streamlines in adjoint solver optimized shape

Figure 1.5 Comparison of Streamlines 
Table 1.3 Comparison of pressure drop of different shapes $(\mathrm{Pa})$

\begin{tabular}{cccc}
\hline & $\begin{array}{l}\text { Total pressure } \\
\text { on inlet }\end{array}$ & $\begin{array}{l}\text { Total pressure } \\
\text { on outlet }\end{array}$ & Pressure drop \\
\hline original & 252.34 & 138.26 & 114.08 \\
\hline Traditional method & 204.22 & 141.13 & 63.09 \\
\hline Adjoint solver & 180.21 & 142.88 & 37.33 \\
\hline
\end{tabular}

Table 1.3 displays the pressure drop values of original shape, traditional method optimized shape and adjoint solver optimized shape. From Figure 1.5and Table 1.3 it can be concluded that to reduce pressure drop, comparing with traditional method, adjoint solver optimized shape has better pressure distribution and flow characteristics as well as smaller pressure drop. As a result adjoint solver optimization has better performance than tradition method[5].

With adjoint solver optimization in optimizing a pipeline, the design can be optimized to achieve better results, while also able to effectively reduce the number of optimization, shorten optimization time.

In practical engineering optimization problems, from the beginning to the final shape, the optimization can be done more than one round. And the more complex the problem is, the more obvious the advantage adjoint solver shows.

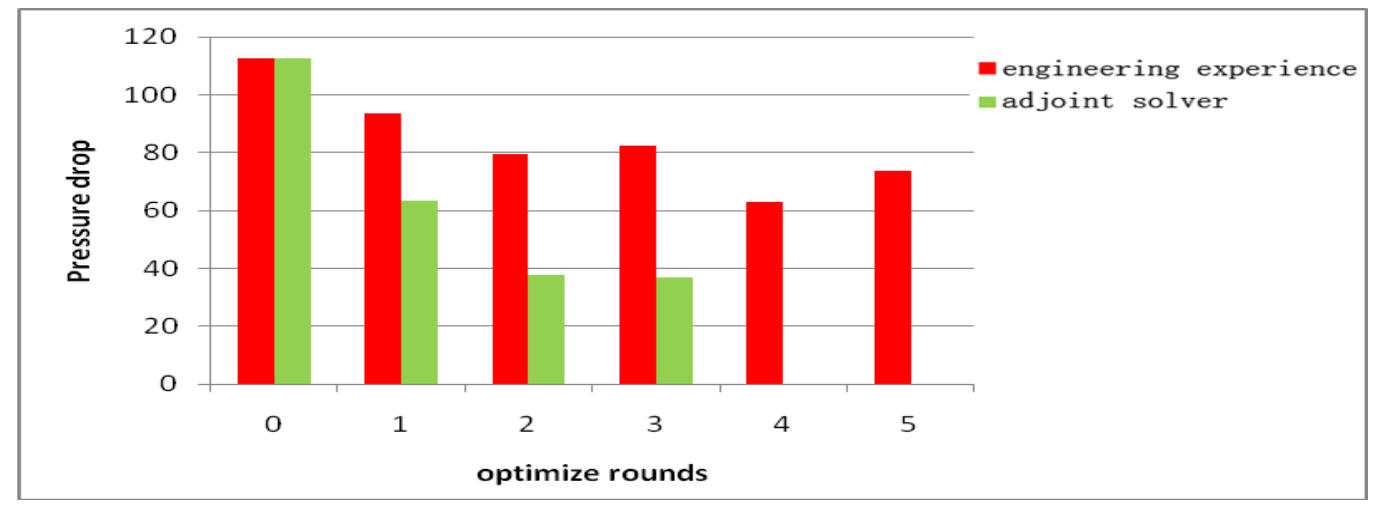

Figure 1.7 Rounds comparison between engineering experience and adjoint solver

Figure 1.7 compares the rounds of traditional method and adjoint solver method. It's shown when using traditional method, although the pressure drop is in a downward trend overall, due to engineering experience has some limitations and blindness, in the optimization process the results are not always better than the previous ones, which leads to more optimization rounds and longer time. Compared with engineering experience, adjoint solver optimization method is based on the sensitivity of the ducts, so each result is based on a relatively small sensitivity further adjustment to the previous one, which leads to more obvious effects and less optimization rounds.

\section{Conclusion}

In this thesis both traditional method and adjoint solver optimization method are used to reduce the pressure drop of a duct, and from both methods an optimized shape is obtained. Compared with traditional method based on engineering experience, adjoint solver optimization method has better performance in optimizing, including higher accuracy and efficiency.

\section{Reference}

[1]. PIRONNEAU O. On optimum shapes in Stokes flow[J]. Journal of Fluid Mechanics, 1973， 59(2): 117 128. 
[2]. Shape Optimization by an Adjoint Solver based on a near-wall Turbulence Model, Sae Technical Papers, 2015

[3]. The research on sensitivity about aerodynamic characteristics of micro-bus based on Morph and DOE [D]. Hunan: Hunan University, 2013.

[4]. Design and numerical simulation of submerged s shaped inlet duct, Electrical Engineering \& Automation , 2013, 2(4):44-51

[5]. Optimization Design of Cold Primary Air Duct based on Fluent. Power System Engineering , 2009 